\title{
Efecto de la empatía sobre el procesamiento cortical temprano y tardío de rostros
}

\author{
Carlos Gantiva ${ }^{1 *}$, Jenny Ricaurte², Andrés Zarabanda², Luz Calderón ${ }^{2}$, Karen Castillo², and \\ Katherine Ortiz ${ }^{2}$ \\ ${ }^{1}$ Department of Psychology, Universidad de Los Andes; ${ }^{2}$ Faculty of Psychology, Universidad de San Buenaventura. Bogotá, Colombia
}

\begin{abstract}
Resumen
Antecedentes: La empatía depende, en gran medida, de la capacidad de procesar las emociones que son expresadas en el rostro de la otra persona. Objetivo: El objetivo del presente estudio fue evaluar las diferencias en el curso del tiempo del procesamiento cognitivo de rostros con expresiones de alegría, neutras e ira en personas con baja y alta empatía. Método: El estudio se llevó a cabo con 60 participantes distribuidos en dos grupos (baja y alta empatía), los cuales observaron rostros con expresiones de alegría, neutras e ira mientras se registraba los potenciales relacionados a eventos $P 100$, N170 y LPP, como indicadores de la atención temprana, codificación del estímulo como un rostro humano y activación y enganche atencional. Resultados: No se encontraron diferencias significativas entre grupos en el componente P100. El potencial N170 fue mayor en el grupo con alta empatía y el LPP fue mayor ante expresiones de ira en el grupo con baja empatía. Conclusiones: Los resultados sugieren que la empatía no tiene un efecto en la respuesta de atención temprana hacia los rostros, pero sí aumenta el reconocimiento del estímulo como un rostro humano y que las personas con baja empatía tienen mayor enganche atencional ante expresiones de ira.
\end{abstract}

Palabras clave: Empatía. Rostros. P100. N170. LPP.

\section{The effect of empathy on early and late cortical face processing}

\begin{abstract}
Background: Empathy depends, to a large extent, on the ability to process the emotions that are expressed in the faces of other people. Objective: The objective of the present study was to evaluate the differences in the time course of cognitive processing of happy, neutral and angry faces in subjects with low and high empathy. Method: The study was carried out with 60 participants divided into two groups (low and high empathy), which observed happy, neutral and angry faces while recording the events related potentials (ERP) P100, N170 and LPP, as indicators of early attention, encoding of the stimulus as a human face and activation and attentional engagement. Results: No significant differences were found between groups in the P100 component. The N170 ERP was higher in the high empathy group and the LPP was higher to angry faces in the
\end{abstract}

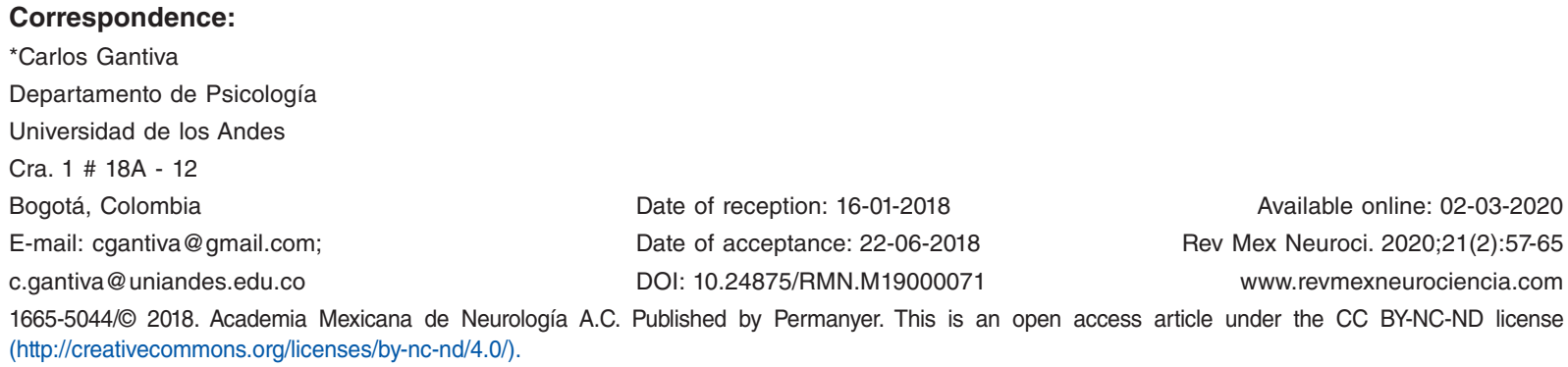

Date of reception: 16-01-2018

Date of acceptance: 22-06-2018

DOI: 10.24875/RMN.M19000071

Available online: 02-03-2020 Rev Mex Neuroci. 2020;21(2):57-65 www.revmexneurociencia.com c.gantiva@uniandes.edu.co 1665-5044/@ 2018. Academia Mexicana de Neurología A.C. Published by Permanyer. This is an open access article under the CC BY-NC-ND license (http://creativecommons.org/licenses/by-nc-nd/4.0/). 
low empathy group. Conclusions: The results suggest that empathy does not have an effect on the response of early attention to faces, but it does increase the recognition of the stimulus as a human face and subjects with low empathy have greater attentional engagement to expressions of anger.

Key words: Empathy. Faces. P100. N170. LPP.

\section{Introducción}

Una de las habilidades fundamentales para la interacción social y la comunicación emocional es la empatía ${ }^{1,2}$, la cual se define como la habilidad para entender y responder a los mensajes emocionales de otros $^{3}$. La empatía está dividida en dos componentes: la empatía afectiva, que es un proceso en donde la percepción de una emoción en otra persona genera una reacción emocional en sintonía con la percibida, y la empatía cognitiva, que es la habilidad para inferir de forma precisa la emoción del otro 4 .

La forma de comunicación más básica de las emociones es por medio de las expresiones faciales ${ }^{5}$, las cuales tienen una función biológica y afectan de forma diferencial al observador. Se ha demostrado que las expresiones faciales generan respuestas de mímica y contagio emocional, por ejemplo, las expresiones faciales de alegría generan activación del músculo cigomático y las expresiones de ira la activación del músculo corrugador ${ }^{6,7}$, incluso cuando las expresiones son vistas por periodos de tiempo muy cortos en donde no hay conciencia de la expresión observada ${ }^{8}$. Sin embargo, el contagio emocional producto de la observación de expresiones emocionales en el rostro se ha encontrado solamente en personas con alta empatía afectiva, las cuales son las que presentan mayor actividad de los músculos cigomático y corrugador ante expresiones de alegría e ira respectivamente ${ }^{9,10}$.

A nivel central el procesamiento de rostros humanos se ha asociado con varios potenciales relacionados a eventos (PRE), principalmente con el componente N170, el cual es un potencial temprano negativo que ocurre en la región parietal-temporal y es un indicador fiable de las etapas tempranas de codificación de las características faciales ${ }^{11,12}$. EI PRE P100 también se ha asociado al procesamiento temprano de rostros, el $\mathrm{P} 100$ es un potencial positivo que sirve como indicador de atención temprana y ocurre en la región occipital-parietal ${ }^{13}$. El P100 aumenta ante la observación de rostros humanos ${ }^{14}$, lo que sugiere la relevancia biológica de las características del rostro. Finalmente, el componente LPP es un potencial positivo tardío que se genera en la región parietal-occipital después de 300 ms y se asocia a activación y atención sostenida hacia diferentes estímulos, entre ellos los rostros humanos, que generan enganche atencional por su relevancia motivacional ${ }^{15}$.

La expresión emocional en el rostro modula la magnitud de algunos PRE, lo que sugiere un aumento o disminución en el procesamiento cognitivo de los rostros, por ejemplo, se ha encontrado que las expresiones de ira y miedo generan un aumento en el LPP ${ }^{16}$. Sin embargo, la evidencia no es concluyente con respecto al efecto de la expresión emocional en los PRE P100 y N170. Por ejemplo, Batty y Taylor ${ }^{11}$ no encontraron diferencias significativas en el P100 ante rostros con distintas expresiones emocionales, sin embargo, Smith, et al. ${ }^{16}$ encontraron que los rostros de miedo generan mayor amplitud del P100 en comparación con los rostros neutrales. Con relación al componente $\mathrm{N} 170$, se ha encontrado que las expresiones de miedo e ira generan mayor amplitud en comparación con las expresiones neutrales ${ }^{11,17}$, aunque otras investigaciones no han encontrado estos mismos datos ${ }^{16}$. Las diferencias en los resultados de los componentes P100 y N170 pueden deberse al uso de distintos estímulos faciales, los cuales pueden variar con relación al nivel de activación temprana que generan en el observador y al nivel de empatía de los participantes.

Pocos estudios han evaluado el efecto de la empatía sobre el procesamiento cortical de los rostros y sus resultados no son concluyentes. Las investigaciones en este campo sugieren que a mayor empatía, mayor respuesta atencional ante los rostros, lo que se refleja en la correlación positiva encontrada entre el nivel de empatía y el aumento en los potenciales N170 y LPP ante rostros con expresiones de alegría, ira, sorpresa, miedo y tristeza ${ }^{18,19}$; sin embargo, los resultados no son sólidos sobre las posibles diferencias entre grupos con baja y alta empatía. Adicionalmente, estas investigaciones no han utilizado rostros con expresiones neutrales, lo que limita sus conclusiones, ya que no se sabe si el aumento en los PRE es producto de las expresiones emocionales o si este fenómeno ocurre ante el rostro humano en general. Tampoco se ha evaluado el procesamiento temprano del rostro por medio del componente P100 en personas con baja y alta empatía, el cual es un indicador de la fase más temprana de atención. 
Por lo anterior, el objetivo del presente estudio fue evaluar las diferencias en el curso del tiempo del procesamiento cognitivo de rostros humanos con expresiones de alegría, neutras e ira en personas con baja y alta empatía, para lo cual se utilizó como indicadores neurofisiológicos los PRE P100, N170 y LPP, como indicadores de la atención temprana, codificación del estímulo como un rostro humano y activación y enganche atencional.

\section{Método}

\section{Participantes}

El estudio se desarrolló con 60 participantes con edades entre 18 y 26 años, los cuales fueron divididos en dos grupos según su puntaje en el índice de reactividad interpersonal ${ }^{20}$ (IRI) y en especial en sus dos escalas principales: toma de perspectiva, la cual mide empatía cognitiva, y preocupación empática, que mide empatía afectiva. Cada grupo estuvo conformado por 30 participantes, el grupo de personas con baja empatía (15 mujeres y 15 hombres, con una edad promedio de 21.10 años, (desviación estándar [DE]: 2.11) y el grupo de personas con alta empatía (14 mujeres y 16 hombres, con una edad promedio de 21.82 años (DE: 2.53). No hubo diferencias significativas entre grupos en la edad de los participantes $\left(\mathrm{t}_{(55)}:-1.16 ; \mathrm{p}=\right.$ $0.25)$, ni en la distribución de hombres y mujeres $\left(\chi_{(1)}^{2}\right.$ : $0.01 ; p=0.88$ ). Los puntajes en las escalas del IRI en cada grupo se presentan en la tabla 1. Se excluyeron participantes con reporte de enfermedades neurológicas, que estuvieran bajo tratamiento farmacológico o con problemas de visión no corregidos. Todos los participantes firmaron el formato de consentimiento informado y el estudio fue aprobado por el Comité de Ética de la institución.

\section{Estímulos}

Se utilizaron 24 imágenes de rostros a color del instrumento NimStim ${ }^{21}$. Ocho mostraban expresiones de alegría, ocho expresiones neutrales y ocho expresiones de ira. Cada expresión emocional fue representada por cuatro hombres y cuatro mujeres. Los modelos utilizados en el experimento fueron aquellos que tenían rasgos latinos (cabello oscuro, ojos café o negros). Para cada una de las expresiones emocionales se utilizaron las imágenes de los mismos modelos, con el objetivo de evitar sesgos de atractivo físico en alguna de las emociones. Los estímulos fueron presentados
Tabla 1. Puntajes promedio (desviación estándar) en las escalas del índice de reactividad interpersonal (IRI) en el grupo de personas con baja y alta empatía

\begin{tabular}{|l|c|c|c|}
\hline Escala del IRI & $\begin{array}{c}\text { Baja } \\
\text { empatía }\end{array}$ & $\begin{array}{c}\text { Alta } \\
\text { empatía }\end{array}$ & p \\
\hline Toma de perspectiva & $15.39(4.48)$ & $19.00(3.90)$ & 0.003 \\
\hline Escala de fantasía & $12.64(4.37)$ & $15.48(5.38)$ & 0.034 \\
\hline $\begin{array}{l}\text { Preocupación } \\
\text { empática }\end{array}$ & $13.39(3.15)$ & $20.27(1.90)$ & $<0.001$ \\
\hline $\begin{array}{l}\text { Estrés personal por } \\
\text { empatía }\end{array}$ & $11.21(5.31)$ & $11.44(4.85)$ & 0.806 \\
\hline Total IRI & $52.64(12.02)$ & $66.20(10.85)$ & $<0.001$ \\
\hline
\end{tabular}

en una pantalla plana de 19 pulgadas sobre un fondo negro localizada a $60 \mathrm{~cm}$ aproximadamente del participante. Cada imagen tuvo un tamaño de $480 \times 480$ píxeles.

Cada estímulo se presentó dos veces con la restricción de no mostrar dos veces seguidas una misma expresión emocional. Se organizaron cuatro órdenes de presentación de estímulos diferentes, los cuales fueron distribuidos de forma equilibrada entre todos los participantes. En general el experimento tuvo un total de 48 ensayos. Cada uno inició con una pantalla negra y una cruz de fijación «+» en el centro durante $500 \mathrm{~ms}$, posteriormente aparecía el rostro durante 2 segundos y finalizaba con un intervalo entre estímulos variable (pantalla negra) entre 3 y 5 segundos. La presentación de los estímulos se programó en el software E-Prime 2.0 (Psychology Software Tools, PA, EE.UU.).

\section{Registro del electroencefalograma}

La actividad electroencefalográfica (el EEG) se registró de forma continua mediante un equipo BioSemi ActiveTwo de 32 canales (BioSemi, Amsterdam, Países Bajos). La ubicación de los electrodos se hizo por medio de un gorro con las coordenadas del sistema internacional 10/20. Durante la adquisición de los datos, la tasa de muestreo fue de $1024 \mathrm{~Hz}$, cada canal se referenció al promedio de la actividad de los 32 canales y se mantuvo la impedancia por debajo de $20 \mathrm{k} \Omega$. Para identificar los parpadeos y movimientos oculares se registró el electrooculograma por medio de dos electrodos ubicados a un centímetro debajo de la pupila del ojo derecho para los movimientos verticales y otro ubicado a un centímetro del canto del ojo izquierdo para los movimientos horizontales. 


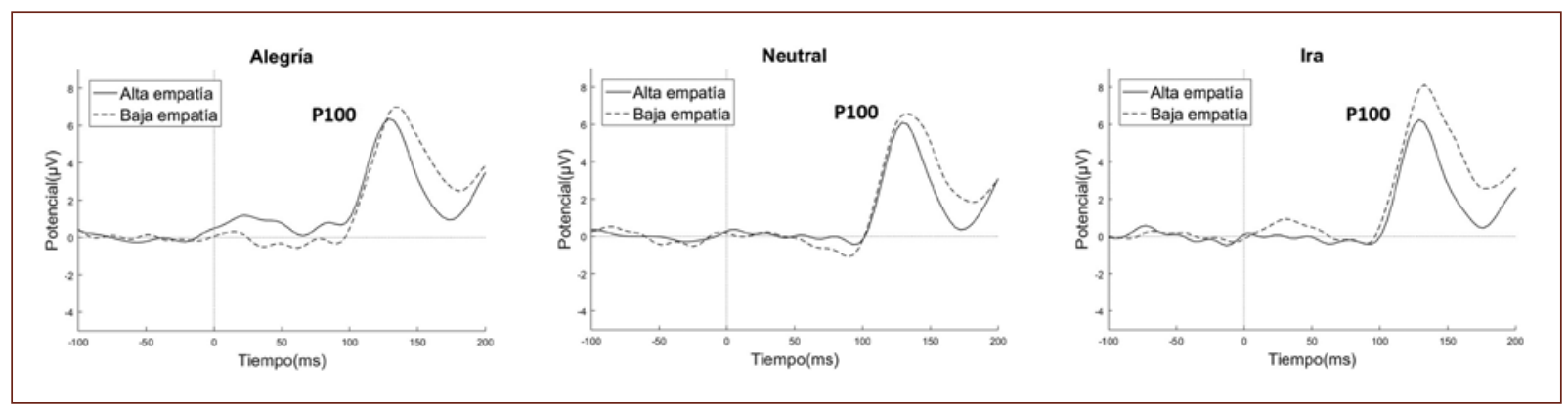

Figura 1. Grand average del potencial relacionado a eventos $\mathrm{P} 100$ en personas con baja y alta empatía ante rostros alegres, neutrales y con ira. Ubicación P03, P04, 01, $0 z$ y 02.

\section{Procesamiento de los datos del EEG}

La señal del EEG se procesó en Matlab a través del toolbox EEGLab ${ }^{22}$ y de scripts diseñados específicamente para el estudio. Inicialmente se disminuyó la tasa de muestreo a $256 \mathrm{~Hz}$ y se aplicó un filtro de banda entre 0.1 y $40 \mathrm{~Hz}$. Se segmentó el registro en intervalos de $1,100 \mathrm{~ms}$ (100 ms preestímulo y $1,000 \mathrm{~ms}$ postestímulo) y se corrigieron los artefactos producto de los movimientos oculares con el algoritmo de Gratton, Coles y Donchin ${ }^{23}$. Se identificaron los valores extremos $(+/-70 \mu \mathrm{V})$ en cada canal y se rechazaron los ensayos que tuvieran cuatro o más canales con estos valores; en total se eliminó el $3.8 \%$ de los ensayos.

Se analizaron la amplitud y la latencia de los PRE P100 y N170 en las regiones en donde la actividad suele ser mayor ${ }^{16,24}$. El P100 se registró en una ventana de tiempo entre 100 a 200 ms en la región parietal-occipital y occipital (electrodos $\mathrm{PO} 3, \mathrm{PO} 4, \mathrm{O} 1, \mathrm{Oz}$ y O2). El N170 se registró en la región parietal (electrodos $\mathrm{P} 7$ y P8), con una ventana de tiempo entre 150 a $250 \mathrm{~ms}$. El potencial LPP se analizó por medio del área bajo la curva $(A B C)$, debido a que al ser un potencial lento no se identifica un pico claro, lo que disminuye la validez de los análisis de amplitud y latencia ${ }^{24}$. El LPP se registró en las zonas parietal y parietal-occipital (electrodos P3, Pz, P4, PO3 y PO4) en una ventana de tiempo entre 300 y $700 \mathrm{~ms}$.

\section{Medidas de autorreporte}

Para la división del grupo de participantes en personas con baja y alta empatía se utilizó la versión del $|\mathrm{IR}|^{20}$ validada en Colombia ${ }^{25}$. EI IRI es un instrumento compuesto por 28 ítems, está dividido en cuatro subescalas que miden empatía cognitiva y empatía afectiva. Las dos escalas principales del IRI son «toma de perspectiva» (empatía cognitiva) y «preocupación empática» (empatía afectiva).

Para la evaluación subjetiva de la emoción se utilizó el maniquí de autoevaluación (Self-Assessment Manikin [SAM]). EI SAM es un instrumento desarrollado por Bradley y Lang ${ }^{26}$, para evaluar las dimensiones de la emoción (valencia, arousal y dominancia). El SAM está conformado por tres escalas, una para cada dimensión, las cuales utilizan cinco figuras humanoides que indican diferentes niveles de intensidad y niveles intermedios entre cada figura. El puntaje en cada escala va de 1 a 9. El SAM ha mostrado niveles apropiados de validez en población colombiana ${ }^{27}$.

\section{Análisis estadístico}

El análisis de los potenciales P100, N170 y LPP se hizo a través de un ANOVA mixta de medidas repetidas, con el factor intersujeto "grupo" (baja y alta empatía) y con los factores intrasujeto «emoción» (alegría, neutral e ira) y «ubicación» (electrodos de cada PRE). El análisis de las medidas subjetivas de la emoción (valencia, arousal y dominancia) se hizo mediante un ANOVA mixto de medidas repetidas $2 \times 3$, con el factor grupo como variable intersujeto y la emoción como variable intrasujeto. Se aplicó la corrección Greenhouse-Geisser en todos los casos para corregir cualquier violación de la esfericidad en los datos. La comparación de pares post hoc se hizo con la corrección Bonferroni. El nivel de significancia para todos los análisis fue de $p<0.05$ y se reporta el tamaño del efecto ${ }^{28}\left(\eta_{p}{ }^{2}\right.$; pequeño $\geq 0.01$, medio $\geq 0.06$ y grande $\geq 0.14$ ). Todos los análisis fueron realizados en el programa estadístico SPSS ${ }^{\circledR} 20.0$ 


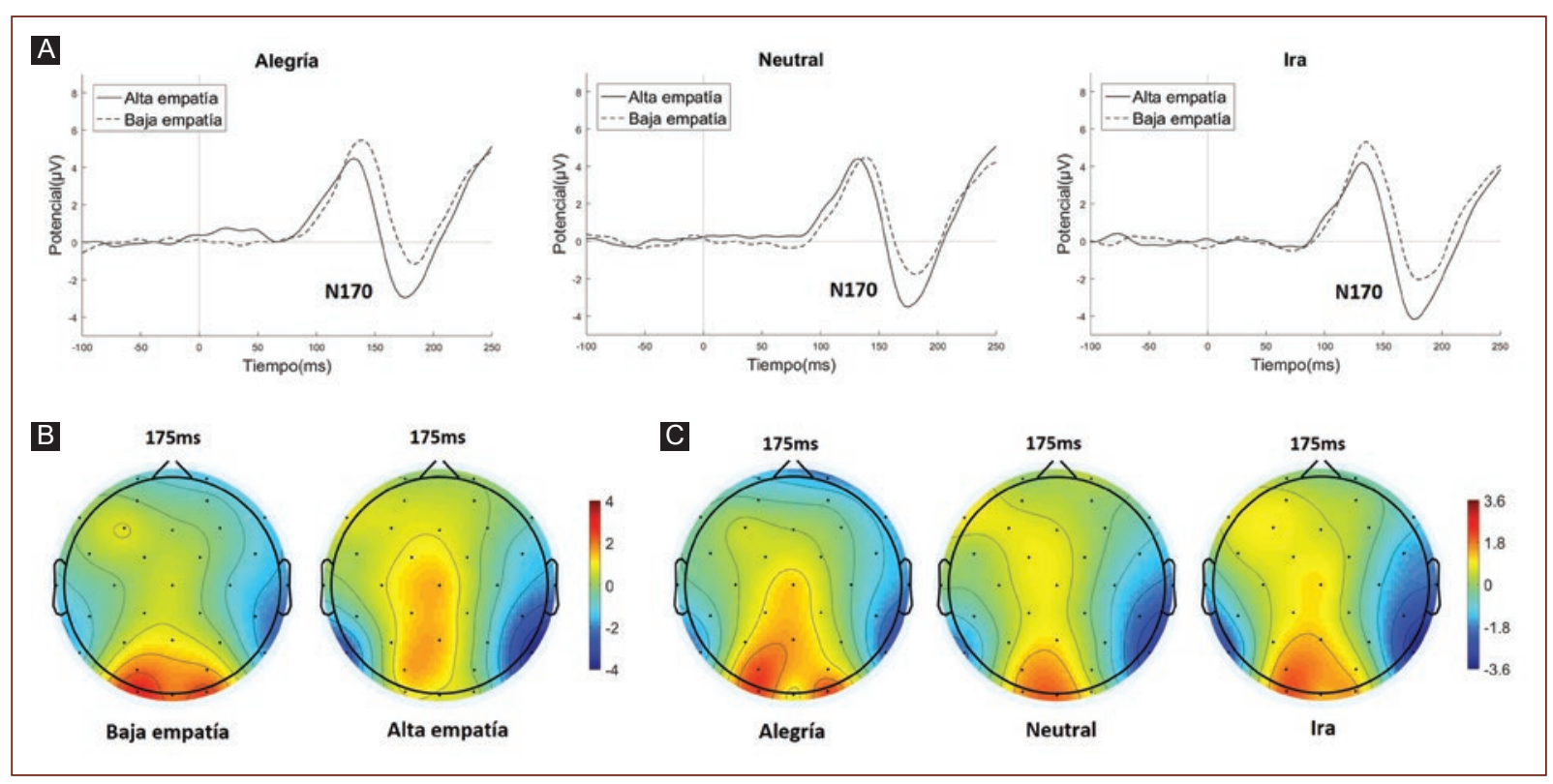

Figura 2. A: grand average del potencial relacionado a eventos N170 en personas con baja y alta empatía ante rostros alegres, neutrales y con ira. Ubicación P7 y P8. B: mapas topográficos de la actividad cortical a los 175 ms para cada grupo. C: mapas topográficos de la actividad cortical a los $175 \mathrm{~ms}$ para expresiones de alegría, neutrales e ira.

\section{Resultados}

\section{P100}

Con respecto a la amplitud del potencial P100, el ANOVA reveló un efecto principal significativo para ubicación $\left(F_{(4,232)}: 36.06 ; p<0.001 ; \eta_{p}^{2}: 0.38\right)$. El P100 fue mayor en la región occipital $(\mathrm{O} 2, \mathrm{Oz}$ y $\mathrm{O} 1)$, en comparación con la región parietal-occipital (PO3 y $\mathrm{PO} 4$ ) (todas las $p<0.001)$. No se encontró ningún otro efecto principal o de interacción significativos (todas las $p>0.11$ ). Con relación a la latencia del P100 no se encontró ningún efecto significativo (todas las $p>0.09$ ) (Fig. 1).

\section{N170}

El ANOVA para la amplitud del potencial evocado N170 encontró un efecto principal significativo para grupo $\left(F_{(1.58)}: 5.05 ; p=0.02 ; \eta_{p}^{2:} 0.08\right)$ y emoción

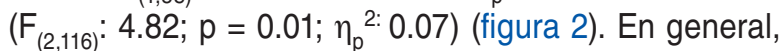
la amplitud del N170 fue mayor (más negativo) en el grupo con alta empatía en comparación con el grupo con baja empatía y las expresiones de ira generaron una mayor amplitud del N170 en comparación con las expresiones de alegría $(p=0.008)$. No hubo otros efectos significativos (todas las $p>0.17$ ).

En relación con la latencia del N170, el ANOVA reveló un efecto principal significativo para ubicación
$\left(F_{(1,58)}: 6.00 ; p=0.01 ; \eta_{p}^{2:} 0.09\right)$, la latencia en el hemisferio derecho (electrodo P8) fue menor que en el hemisferio izquierdo (electrodo P7). No se encontró ningún otro efecto principal o de interacción significativo (todas las $p>0.22$ ).

\section{LPP}

El ANOVA para el ABC del potencial evocado LPP encontró un efecto principal y de interacción significa-

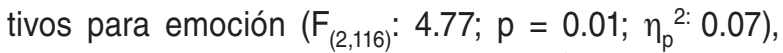

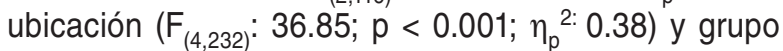
$x$ emoción $\left(F_{(2,116)}: 4.61 ; p=0.01 ; \eta_{p}^{2:} 0.07\right)$ (Fig. 3). En general, los rostros de ira y alegría generaron mayor LPP que los rostros neutrales, lo que se refleja en una tendencia cuadrática y significativa de los da-

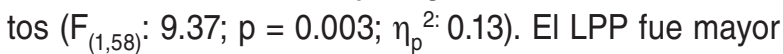
en los electrodos $\mathrm{PO} 3$ y PO4 en comparación con P3, Pz y P4 (todas las $p<0.001$ ). Con respecto a la interacción grupo $x$ emoción, se encontró que en el grupo de participantes con baja empatía el LPP fue mayor ante los rostros de ira en comparación con los rostros neutrales y alegres (ambas $p<0.05$ ).

\section{Medidas de autorreporte}

En la tabla 2 se muestran los puntajes en valencia, arousal y dominancia para cada una de las 


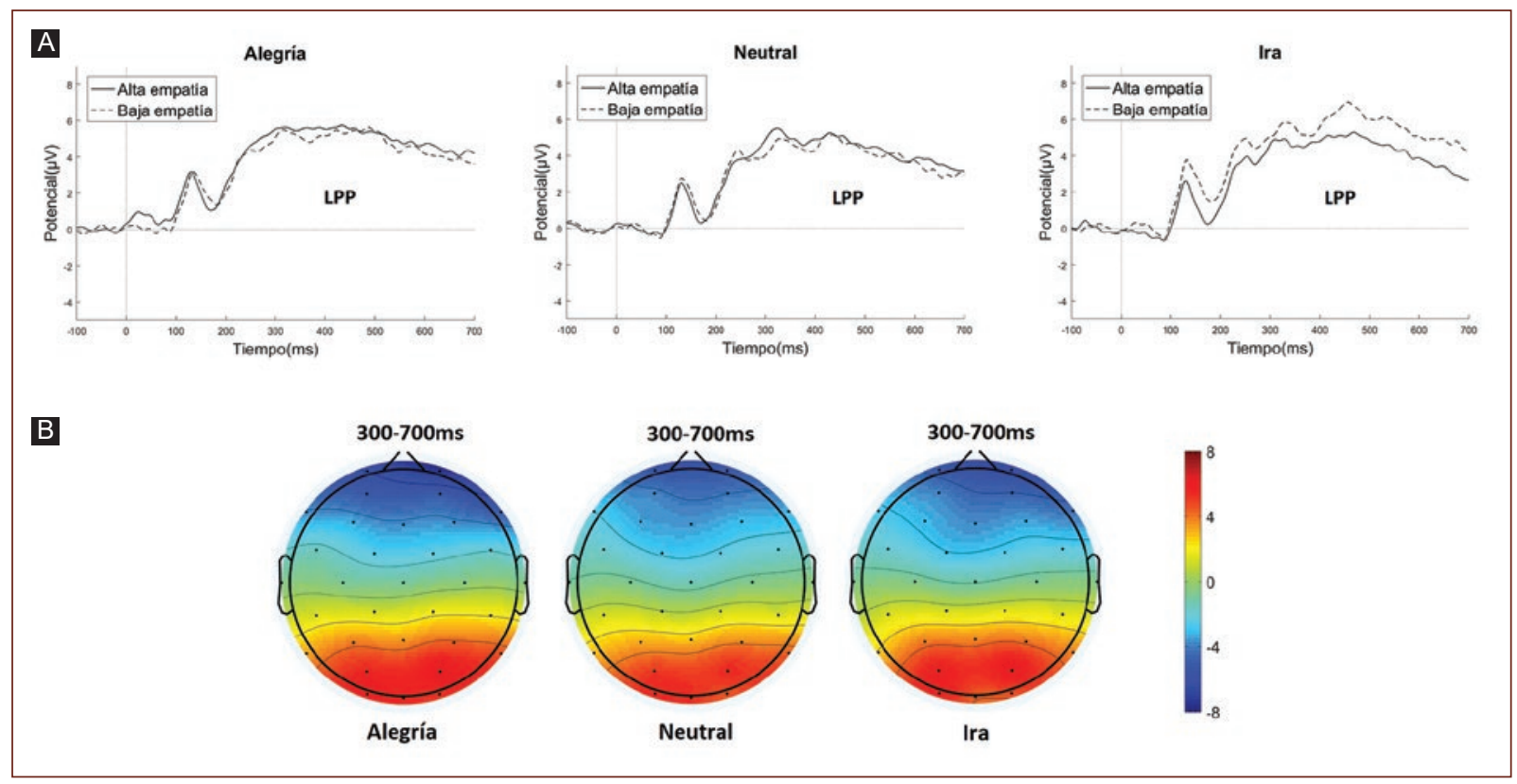

Figura 3. A: grand average del potencial relacionado a eventos LPP en personas con baja y alta empatía ante rostros alegres, neutrales y con ira. Ubicación P3, Pz, P4, P03 y P04. B: mapas topográficos de la actividad cortical promedio de los 300 a 700 ms para expresiones de alegría, neutrales e ira.

expresiones emocionales. El ANOVA para la dimensión de valencia identificó un efecto principal significativo para emoción $\left(F_{(2,110)}: 82.28 ; p<0.001 ; \eta_{p}^{2:} 0.59\right)$. Las expresiones de ira generaron estados afectivos negativos, las expresiones de alegría estados afectivos positivos y las expresiones neutrales estados afectivos neutrales (todas las $p<0.001$ ). No se encontraron otros efectos principales ni de interacción significativos (todas las $p>0.62$ ). Para la dimensión de arousal el ANOVA encontró un efecto principal significativo para emoción $\left(F_{(2,110)}: 6.56 ; p=0.002 ; \eta_{p}^{2:} 0.10\right)$. Las expresiones de ira generaron mayor activación que las expresiones neutrales $(p=0.002)$. No se encontraron otros efectos principales ni de interacción significativos (todas las $p>0.29$ ). Por último, para la dimensión de dominancia, el ANOVA nuevamente encontró un efecto principal significativo para emoción $\left(\mathrm{F}_{(2,110)}\right.$ : 3.36 ; $\left.p=0.04 ; \eta_{p}^{2:} 0.05\right)$. Las expresiones de ira generaron menor percepción de control en comparación con las expresiones de alegría $(p=0.05)$. No se encontraron otros efectos principales ni de interacción significativos (todas las $p>0.71$ ).

\section{Discusión}

El presente estudio utilizó técnicas neurofisiológicas para evaluar en el curso del tiempo las posibles
Tabla 2. Puntajes promedio (desviaciones estándar) en valencia, arousal y dominancia ante cada expresión emocional

\begin{tabular}{|l|c|c|c|}
\hline & Alegría & Neutral & Ira \\
\hline Valencia & $6.38(1.52)$ & $4.68(1.01)$ & $3.74(1.47)$ \\
\hline Arousal & $4.03(1.73)$ & $3.60(1.89)$ & $4.28(1.83)$ \\
\hline Dominancia & $7.18(1.71)$ & $7.00(1.87)$ & $6.72(1.76)$ \\
\hline
\end{tabular}

diferencias en el procesamiento cognitivo de rostros humanos con expresiones de alegría, neutras e ira en personas con baja y alta empatía. Los resultados no mostraron diferencias significativas en el componente P100, lo que sugiere respuestas similares de atención temprana hacia los rostros en ambos grupos, sin embargo, sí se observaron diferencias en el procesamiento posterior, el potencial $\mathrm{N} 170$ es mayor en las personas con alta empatía, lo que sugiere una mayor codificación o reconocimiento de las características faciales. También se identificaron diferencias en el enganche atencional en las personas con baja empatía, las cuales respondieron con mayor atención sostenida ante las expresiones de ira.

La ausencia de diferencias significativas entre grupos en el P100 sugiere que la respuesta de atención inicial hacia los rostros no se ve influenciada por el nivel de empatía de las personas, lo que coincide con 
las conclusiones del estudio de Herrmann, et al. ${ }^{14}$ relacionadas con la alta relevancia biológica y motivacional de los rostros, los cuales generan una mayor respuesta de atención temprana en comparación con otros tipos de estímulos. En conjunto, estos resultados sugieren que la primera respuesta atencional hacia el rostro es un proceso muy básico mediado más por variables de orden evolutivo que de aprendizaje individual. Tampoco hubo diferencias en el P100 con relación al tipo de expresión emocional, estos mismos resultados se han reportado previamente ${ }^{11}$, lo que indica que la primera respuesta atencional hacia el rostro no parece estar modulada por la emoción expresada.

Los resultados en el componente N170 sugieren un mayor procesamiento atencional y de reconocimiento del rostro humano en las personas con alta empatía. Resultados similares han sido reportados previamente $^{18,19}$, lo que indica que el procesamiento detallado del rostro (sus características, rasgos, expresiones) aumenta la posibilidad del reconocimiento emocional y la generación posterior de un estado afectivo en sintonía con la expresión observada, es decir, una respuesta de empatía afectiva. Este mecanismo ha sido previamente descrito en los estudios sobre contagio emocional ${ }^{29,30}$ y empatía ${ }^{9}$, en donde el proceso inicia con la percepción del rostro del otro individuo, continua con la respuesta de mímica facial que sirve como mecanismo de retroalimentación para la propia persona y la induce a un estado emocional similar, lo que finaliza en una respuesta de empatía afectiva.

Este proceso a nivel neurobiológico estaría soportado por el sistema de las neuronas espejo que valida la teoría de la simulación como mecanismo para el desarrollo de la empatía ${ }^{31,32}$. Los estudios con neuroimagen han mostrado que el sistema de las neuronas espejo en los humanos se encuentra conformado por una red compleja de áreas visuales en las regiones occipital, parietal y temporal y dos regiones predominantemente motoras (parte anterior del lóbulo parietal inferior y el sector del lóbulo frontal conformado por el córtex premotor ventral más la región posterior del giro frontal

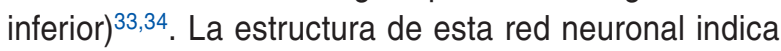
que gran parte del proceso se da en la región parietal, en donde el potencial N170 ocurre con mayor amplitud $^{11,16,24}$ y en donde se registró el potencial N170 en este estudio (electrodos P7 y P8), siendo menor la latencia en el hemisferio derecho.

Los resultados con relación al efecto de la expresión emocional en el PRE N170 indican que las expresiones de ira aumentan su amplitud, lo que probablemente esté relacionado con una mayor relevancia conductual de la ira en comparación con la alegría. Estudios previos han mostrado que las expresiones de ira por sí solas logran activar el sistema motivacional defensivo, reflejado en la potenciación del reflejo de sobresalto, mientras que las expresiones de alegría, sin un contexto que las acompañe, no lo logran inhibiri ${ }^{35}$. Esta diferencia se debe a que históricamente las expresiones de ira han evolucionado como señales de peligro en sí mismas y el organismo se prepara para responder automáticamente ante ellas ${ }^{36}$.

Los resultados en el LPP indican, como era esperable, un mayor enganche atencional ante las expresiones de ira y alegría en comparación con los rostros neutrales. Múltiples estudios han mostrado que el LPP es sensible al arousal generado por los estímulos, lo que indica que se produce una mayor respuesta de atención sostenida (mayor magnitud del LPP) hacia estímulos emocionalmente relevantes y un menor LPP ante estímulos neutrales ${ }^{15,16}$.

También se encontró que las personas con baja empatía respondieron con un mayor LPP ante las expresiones de ira en comparación con las demás expresiones. Estos resultados no fueron encontrados en las investigaciones previas en donde se estudió la relación entre empatía y LPP ${ }^{18,19}$, sin embargo, puede estar relacionado con la mayor relevancia conductual de las expresiones de ira ${ }^{35,36}$, que lleva a que incluso las personas con baja empatía centren su atención en esta expresiones. Este resultado también podría ser coherente con la hipótesis de la relación entre empatía, regulación emocional y agresividad ${ }^{37}$, ya que si las personas con baja empatía se enganchan atencionalmente con las expresiones de ira podrían tener mayores dificultades para regularse emocionalmente y generar, posteriormente, una respuesta de agresión.

Finalmente, los resultados en las medias de autorreporte no mostraron diferencias significativas entre grupos en ninguna de las dimensiones de la emoción (valencia, arousal y dominancia), lo que sugiere que la percepción de la experiencia emocional puede ser similar en personas con baja y alta empatía cuando observan rostros alegres, neutros y con ira, es decir, que se experimenta, por ejemplo, una valencia positiva ante los rostros alegres y aversiva ante los rostros con ira en ambos grupos. Sin embargo, la empatía es la capacidad para entender y experimentar una emoción en sintonía con la observada ${ }^{3,4}$, por lo cual se requiere una experiencia emocional más precisa que solamente la 
percepción de la valencia (apetitiva o aversiva), el arousal y la dominancia, por lo cual, las dimensiones de la emoción no son, necesariamente, indicadores precisos de empatía.

Los resultados del presente estudio deben analizarse tomando en cuenta varias limitaciones. Primero, solamente se estudiaron tres expresiones faciales (alegría, neutra e ira), por lo cual futuras investigaciones deberán analizar las posibles diferencias entre las demás emociones. Segundo, la muestra estuvo conformada por personas con baja y alta empatía, sin embargo, no hubo un grupo que estuviera ubicado en niveles intermedios de empatía, el análisis de este tercer grupo permitirá comprender mejor el procesamiento cognitivo de las expresiones emocionales. Finalmente, el estudio aborda la empatía afectiva como un solo constructo con dos polos (baja y alta), sin embargo, estudios recientes han propuesto que la empatía afectiva podría ser dividida en dos tipos (resonancia afectiva y disonancia afectiva) ${ }^{38}$. La primera hace referencia al concepto tradicional de empatía afectiva y la segunda a personas que sienten lo opuesto a lo que observan (p. ej., placer ante el dolor ajeno o rabia ante la felicidad del otro), futuros estudios podrán utilizar esta nueva forma de evaluar la empatía afectiva en conjunto con técnicas neurofisiológicas para investigar el procesamiento de rostros.

\section{Conclusiones}

En conjunto, los resultados del presente estudio sugieren que la empatía no tiene un efecto en la respuesta de atención inicial ante los rostros humanos (P100), sin embargo, sí tiene un efecto sobre el reconocimiento de las características del rostro (mayor N170 en personas con alta empatía). Finalmente, las personas con baja empatía generan mayor enganche atencional (mayor LPP) hacia las expresiones de ira.

\section{Financiamiento}

Esta investigación se ha realizado dentro del proyecto CHS 012-010, financiado por la Universidad de San Buenaventura, sede Bogotá (Colombia).

\section{Conflicto de intereses}

Los autores declararan no tener conflictos de interés.

\section{Responsabilidades éticas}

Protección de personas y animales. Los autores declaran que los procedimientos seguidos se conformaron a las normas éticas del comité de experimentación humana responsable y de acuerdo con la Asociación Médica Mundial y la Declaración de Helsinki.

Confidencialidad de los datos. Los autores declaran que en este artículo no aparecen datos de pacientes.

Derecho a la privacidad y consentimiento informado. Los autores declaran que en este artículo no aparecen datos de pacientes.

\section{Bibliografía}

1. Gutiérrez M, Escartí A, Pascual C. Relaciones entre empatía, conducta prosocial, agresividad, autoeficacia y responsabilidad personal y social de los escolares. Psicothema. 2011;23(1):13-9.

2. Light SN, Moran ZD, Swander L, Le V, Cage B, Burghy C, et al. Electromyographically assessed empathic concern and empathic happiness predict increased prosocial behavior in adults. Biol Psychol. 2015;104:116-29.

3. Decety J, Jackson PL. The functional architecture of human empathy. Behav Cogn Neurosci Rev. 2004;3(2):71-100.

4. Decety J. Dissecting the neural mechanisms mediating empathy. Emot Rev.2011;3(1):92-108.

5. Darwin C. The expression of emotion in man and animals. London: Murray; 1872.

6. Dimberg U. Facial reactions to facial expressions. Psychophysiology. 1982;19:643-7.

7. Lundquist LO, Dimberg U. Facial expressions are contagious. J Psychophysiol. 1995:9:203-11.

8. Dimberg U, Thunberg M, Elmehed K. Unconscious facial reactions to emotional facial expressions. Psychol Sci. 2000;11(1):86-9.

9. Dimberg U, Andréasson $P$, Thunberg M. Emotional empathy and facial reactions to facial expressions. J Psychophysiol. 2011;25(1):26-31.

10. Dimberg U, Thunberg M. Empathy, emotional contagion, and rapid facial reactions to angry and happy facial expressions. Psych Journal. 2012;1(2):118-27.

11. Batty M, Taylor MJ. Early processing of the six basic facial emotional expressions. Cognitive Brain Res. 2003;17(3):613-20.

12. Olivares El, Saavedra C, Iglesias J. Potenciales evocados como marcadores neurofisiológicos de la percepción y el reconocimiento de caras. Rev Lat Psic. 2012;44(2):27-38.

13. Luck S. Multiple mechanisms of visual-spatial attention: recent evidence from human electrophysiology. Behav Brain Res. 1995;71(1):113-23.

14. Herrmann MJ, Ehlis AC, Ellgring H, Fallgatter AJ. Early stages (P100) of face perception in humans as measured with event-related potentials (ERPs). J Neural Transm. 2005;112(8):1073-81.

15. Cuthbert BN, Schupp HT, Bradley MM, Birbaumer N, Lang PJ. Brain potentials in affective picture processing: covariation with autonomic arousal and affective report. 凶Biol Psychol. 2000;52(2):95-111.

16. Smith E, Weinberg A, Moran T, Hajcak G. Electrocortical responses to NIMSTIM facial expressions of emotion. Int J Psychophysiol. 2013;88: 17-25.

17. Bonilla F, Leongómez J. Efectos en la amplitud y la latencia del componente N170 ante la presentación de rostros emocionales de ira y miedo. Psychol. 2017;11(1):39-48.

18. Choi D, Nishimura T, Motoi M, Egashira Y, Matsumoto R, Watanuki S. Effect of empathy trait on attention to various facial expressions: evidence from N170 and late positive potential (LPP). J Physiol Anthropol. 2014;33:18.

19. Choi D, Watanuki S. Effect of empathy trait on attention to faces: an event-related potential (ERP) study. J Physiol Anthropol. 2014;33:4.

20. Davis MH. A multidimensional approach to individual differences in empathy [Internet]. JSAS Catalog of Selected Documents in Psychology; 1980. Disponible en: http://www.uv.es/ friasnav/Davis_1980.pdf

21. Tottenham N, Tanaka JW, Leon AC, McCarry T, Nurse M, Hare TA, et al. The NimStim set of facial expressions: judgments from untrained research participants. Psychiatry Res. 2009;168(3):242-9.

22. Delorme A, Makeig S. EEGLAB: an open source toolbox for analysis of single-trial EEG dynamics including independent component analysis. J Neurosci Methods. 2004;134(1):9-21.

23. Gratton $G$, Coles MG, Donchin E. A new method for offline removal of ocular artifact. Electroencephalogr Clin Neurophysiol. 1983;55:468-84. 
24. daSilva E, Crager K, Puce A. On dissociating the neural time course of the processing of positive emotions. Neuropsychologia. 2016;83:123-37.

25. Pineda DA, Aguirre-Acevedo DC, Trujillo N, Valencia AM, Pareja A, Tobón $\mathrm{C}$, et al. Dimensiones de la empatía en excombatientes del conflicto armado colombiano utilizando una escala estandarizada. Rev Colomb Psiquiatr. 2013;42(1):9-28.

26. Bradley MM, Lang PJ. Measuring emotion: The self-assessment manikin and the semantic differential. J Behav Ther Exp Psychiatry. 1994;25:49-59.

27. Gantiva C, Guerra P, Vila J. Validación colombiana del sistema internacional de imágenes afectivas: evidencias del origen transcultural de la emoción. Acta Colomb Psicol. 2011;14(2):103-11.

28. Cohen J. Statistical power analysis for the behavioral sciences $\left(2^{\text {nd }}\right.$ ed. $)$. Hillsdale, NJ: Erlbaum; 1988.

29. Hatfield E, Cacioppo JT, Rapson R. Primitive emotional contagion. En Clark MS (ed.). Review of personality and social psychology: Vol. 14 Emotion and social behavior. Newbury Park, CA: Sage; 1992. pp. 151-77.

30. MacDonald A. I feel your pain (and joy): New theories about empathy. Brain Work. 2003;13(4):1-3.
31. Gallese V. The "shared Manifold" hypothesis. From mirror neurons to empathy. J Conscious Stud. 2001;8(5-6):33-50.

32. Gallese V, Goldman A. Mirror neurons and the simulation theory of mind-reading. Trends Cogn Sci. 1998;2(12):493-501.

33. Acharya S, Shukla S. Mirror neurons: Enigma of the metaphysical modular brain. J Nat Sci Biol Med. 2012;3(2):118-24.

34. Rizzolatti G, Craighero L. The mirror-neuron system. Annu Rev Neurosci. $2004 ; 27: 169-92$.

35. Alpers GW, Adolph D, Pauli P. Emotional scenes and facial expressions elicit different psychophysiological responses. Int J Psychophysiol. 2011;80(3):173-81.

36. Dimberg U, Öhman A. Behold the wrath: Psychophysiological responses to facial stimuli. Motiv Emot. 1996;20(2):149-82.

37. Vachon DD, Lynam DR, Johnson JA. The (non) relation between empathy and aggression: Surprising results from a meta-analysis. Psychol Bull. 2014;140(3):751-73.

38. Vachon DD, Lynam DR. Fixing the problem with empathy: Development and validation of the affective and cognitive measure of empathy. Assessment. 2016;23(2):135-49. 